\title{
OPTIC DISC AND RETINAL FINDINGS IN OPTICAL COHERENCE TOMOGRAPHY IN IDIOPATHIC INTRACRANIAL HYPERTENTION
}

Alaa Mohamed Fadel, Ihab Mohamed Ossman, Mirette Magdy Hakim Rezkallah Kars Department of Ophthalmology, Faculty of Medicine, University of Alexandria.

INTRODUCTION
Idiopathic intracranial hypertension (IIH) is a condition of increased intracranial pressure
of unknown cause. It can have the devastating effect of permanent vision loss seen in $25 \%$
of patients from secondary optic neuropathy if patients are not treated in a timely and
appropriate manner.
The neuro-ophthalmic findings in idiopathic intracranial hypertension patients are mainly
visual loss and papilledema. Papilledema is caused by increased ICP and is a cardinal sign
of IIH. Although OCT is frequently used in clinical practice to evaluate thinning of the
retinal nerve fiber layer (RNFL) due to optic nerve injury from a variety of causes, using
OCT to quantify papilledema is relatively new. Evaluating OCT findings in IIH patients
and comparing it with clinical and perimetric findings could be useful.

\section{AIM OF THE WORK}

In this study we employed OCT to evaluate its value in detecting structural changes in the optic nerve head and the retina of patients with IIH to determine if it provides additional help to identify optic disc swelling and correlate the findings with clinical and perimetric evaluation.

\section{SUBJECTS AND METHODS}

A prospective, cross sectional, study was carried out on 20 eyes in referred patients with IIH at the Ophthalmology Department of Alexandria Main University Hospital.

Patients with optic nerve disorders (such as congenital coloboma or microphthalmos), Patillitis, a positive history of 21 ins

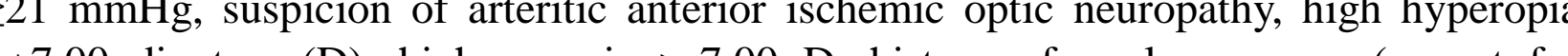
$>+7.00$ diopters (D), high myopia $>-7.00 \mathrm{D}$, hist of uncomplicated cataract surgery), advanced cataract or cloudy media opacity were excluded from our study.

we used the Modified Frisen Scale (MFS) to define the grade of papilledema in each eye and we divided them into subgroups, mild PO (grade 0,1 and 2) $(\mathrm{n}=10)$ and moderate $\mathrm{PO}$ (grade 3$) \mathrm{n}=(5)$ and marked PO (grade 4$)(\mathrm{n}=5)$. We excluded grade 5 cases from our analysis because in those examples, in OCT images the algorithm did fail.

Automated visual field (VF) was done to all patients.

OCT examination included the following acquisition protocols: Optic disc cube centered $\mathrm{ONH}$, Macular Cube centered on the fovea and macular cube centered on the optic nerve head.

\begin{tabular}{|c|c|c|c|c|c|}
\hline & \multicolumn{4}{|c|}{ RESULTS } & \\
\hline \multicolumn{6}{|c|}{ Table 1: Comparison between the three studied groups according to RNFL } \\
\hline RNFL & $\begin{array}{c}\text { Mild } \\
(\mathrm{n}=10)\end{array}$ & $\begin{array}{c}\text { Moderate/ Severe } \\
(\mathbf{n}=\mathbf{1 0})\end{array}$ & $\begin{array}{l}\text { Control } \\
(\mathbf{n}=\mathbf{2 0})\end{array}$ & $\mathbf{F}$ & $\mathbf{p}$ \\
\hline Average & & & & & \\
\hline \begin{tabular}{|l} 
Mean \pm SD. \\
Median \\
(IQR)
\end{tabular} & $\begin{array}{c}121.40 \pm 4.20 \\
120.5 \\
(117.8-125.3) \\
\end{array}$ & $\begin{array}{c}298.30 \pm 6.43 \\
299.0 \\
(294.0-302.0)\end{array}$ & $\begin{array}{c}116.55 \pm 7.13 \\
118.0 \\
(116-120) \\
\end{array}$ & $3004.47 *$ & $<0.001 *$ \\
\hline Sig. bet. Grps & $p_{1}<0$. & $001^{*}, p_{2}=0.135, p_{3}<\mathrm{c}$ & $001^{*}$ & & \\
\hline 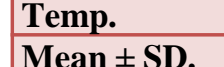 & $68.60 \pm 5.68$ & $2040 \pm 10.45$ & $71.20 \pm 6.96$ & & \\
\hline $\begin{array}{l}\text { Median } \\
\text { (IOR) }\end{array}$ & $\begin{array}{c}68.50 \\
(63.75-74.25)\end{array}$ & $\begin{array}{c}205.0 \\
(194.0-212.75)\end{array}$ & $\begin{array}{c}70.50 \\
(65.5-76.5)\end{array}$ & $1130.49 *$ & $<0.001 *$ \\
\hline Sig. bet. Grps & $\mathrm{p}_{1}<0$. & $001^{*}, \mathrm{p}_{2}=0.661, \mathrm{p}_{3}<\mathrm{C}$ & $001^{*}$ & & \\
\hline $\mid \begin{array}{l}\text { Superior } \\
\text { Mean } \pm \text { SD. }\end{array}$ & $142.60 \pm 5.99$ & $400.30 \pm 10.57$ & $136.45 \pm 6.74$ & & \\
\hline $\begin{array}{l}\text { Median } \\
\text { (IQR) }\end{array}$ & $\begin{array}{c}140.50 \\
(137.75-149.3)\end{array}$ & $\begin{array}{c}403.5 \\
(389.75-408.5)\end{array}$ & $\begin{array}{c}135.0 \\
(131.5-141.5)\end{array}$ & $4341.639 *$ & $<0.001 *$ \\
\hline Sig. bet. Grps & $p_{1}<0$. & $001^{*}, \mathrm{p}_{2}=0.112, \mathrm{p}_{3}<\mathrm{C}$ & $001^{\prime \prime}$ & & \\
\hline Mean \pm SD. & $87.90 \pm 4.53$ & $255.0 \pm 9.49$ & $84.70 \pm 6.06$ & & \\
\hline $\begin{array}{l}\text { Median } \\
\text { (IOR) }\end{array}$ & $\begin{array}{c}88.50 \\
(83.75-92.25)\end{array}$ & $\begin{array}{c}258.50 \\
(246.0-261.0)\end{array}$ & $\begin{array}{c}86.0 \\
(8.5895)\end{array}$ & 2347.79* & $<0.001^{*}$ \\
\hline Sig. bet. Grps & & $001^{*}, \mathrm{p}_{2}=0.448, \mathrm{p}_{3}<\mathrm{C}$ & & & \\
\hline $\mid \begin{array}{l}\text { Inferior } \\
\text { Mean } \pm \text { SD. }\end{array}$ & $147.80 \pm 5.01$ & $346.60 \pm 7.40$ & $142.80 \pm 7.44$ & & \\
\hline $\begin{array}{l}\text { Median } \\
\text { GOOR }\end{array}$ & 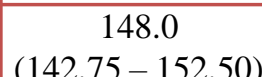 & & 145.50 & $3206.03^{*}$ & $<0.001 *$ \\
\hline \begin{tabular}{|l|l} 
(QQR) \\
Sig. bet. Grps \\
\end{tabular} & $\frac{(142.75-152.50)}{p_{1}<0}$ & $\frac{\mid(3399.7-352.5)}{001^{3}, p_{2}=0.163, p_{3}<0}$ & $\frac{(139-148.5)}{001^{*}}$ & & \\
\hline
\end{tabular}

Table 2: Comparison between the three studied groups according to GCL

\begin{tabular}{|c|c|c|c|c|c|c|}
\hline GCL & $\begin{array}{c}\text { Mild } \\
(\mathbf{n}=\mathbf{1 0})\end{array}$ & $\begin{array}{c}\text { Moderate } \\
(\mathbf{n}=5)\end{array}$ & $\begin{array}{l}\text { Severe } \\
(\mathrm{n}=5)\end{array}$ & $\begin{array}{l}\text { Control } \\
(\mathbf{n}=20)\end{array}$ & H & $\mathbf{p}$ \\
\hline Iean & $88.40 \pm 3.03$ & $78.0 \pm 1.58$ & $68.0 \pm 2.92$ & $89.40 \pm 4.69$ & & \multirow[b]{2}{*}{$<0.001^{*}$} \\
\hline Median(IQR) & $\begin{array}{r}88.5 \\
8601\end{array}$ & 78.0 & 67.0 & 88. & & \\
\hline & 0.934 & $\frac{(17.0-19.0)}{0.002^{*}}$ & $\frac{(00.0-10 .)}{<0.001^{*}}$ & $(85.5-50.5)$ & & \\
\hline g.bet.Gr & $p_{1}=$ & $\mathrm{p}_{2}<0.001^{*}$ & $=0.498$ & & & \\
\hline
\end{tabular}

Table 3: Correlation between MD and grade of papilledema from colored fundus photographs \begin{tabular}{|l|c|c|}
\hline MD vs grade & & \\
\hline Cases (n=20) & $0.733^{*}-$ & $<0.001^{*}$ \\
\hline Mild $(\mathbf{n}=\mathbf{1 0}$ & 0.338 & $0.339)^{*}$ \\
\hline
\end{tabular}

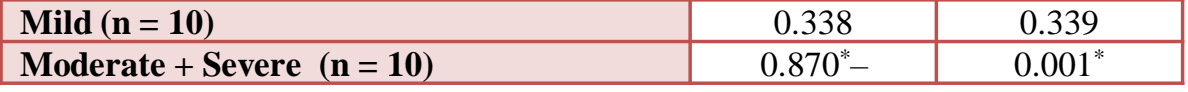

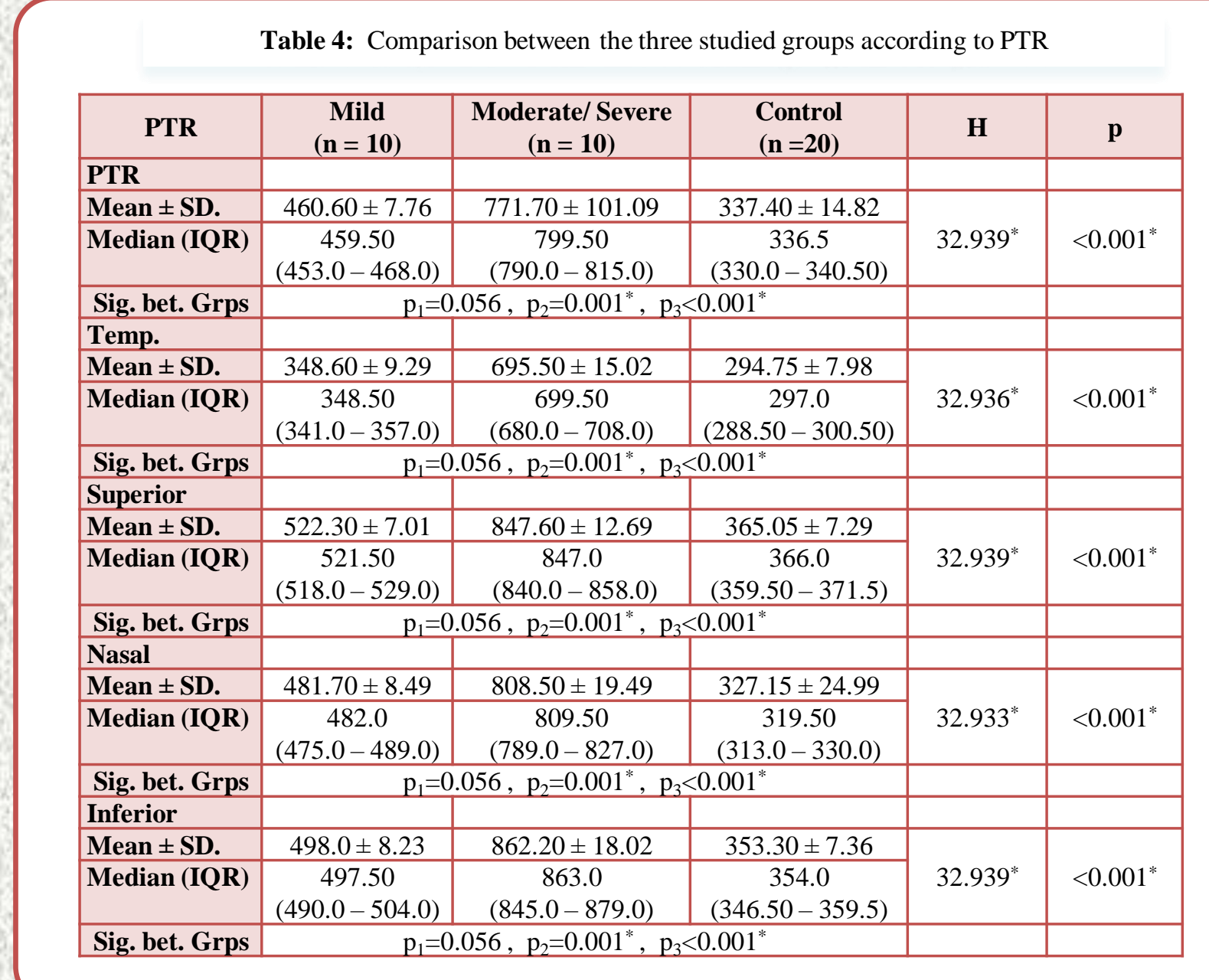

CONCLUSION

Perimetry and clinical evaluation although very effective in papilledem evaluation they have their limitations in evaluating papilledema and OCT can be a helpful additional tool: Although RNFL and PTR thickness were equivalent to document moderate-severe PO, only PTR could differentiate patients with mild PO from controls, Besides, RNFL is not able to detect comitant optic atrophy in the setting of papilledema making it difficult to predict vision loss so in this cases we can rely on GCL as an early predictor of vision loss especially when perimetry is unreliable. 